Original

\title{
Bone marrow-derived mesenchymal stem cells enhance bone marrow regeneration in dental extraction sockets
}

\author{
Takayuki Mashimo ${ }^{1,2)}$, Yukio Sato ${ }^{3)}$, Daisuke Akita4), Taku Toriumi ${ }^{5}$, \\ Shunsuke Namaki6), Yumi Matsuzaki7), Yoshiyuki Yonehara6), and Masaki Honda5) \\ 1)Department of Oral and Maxillofacial Surgery, Juntendo University Faculty of Medicine, Tokyo, Japan \\ 2)Department of Oral and Maxillofacial Surgery, Nihon University School of Dentistry, Tokyo, Japan \\ 3)Department of Emergency and Critical Care Medicine, Keio University School of Medicine, Tokyo, Japan \\ 4)Department of Partial Denture Prosthodontics, Nihon University School of Dentistry, Tokyo, Japan \\ 5)Department of Oral Anatomy, Aichi-Gakuin University School of Dentistry, Nagoya, Japan \\ 6)Department of Clinical Medicine, Nihon University School of Dentistry, Tokyo, Japan \\ 7)Department of Life Science, Shimane University Faculty of Medicine, Izumo, Japan
}

(Received April 27, 2018; Accepted August 15, 2018)

\begin{abstract}
Bone marrow-derived mesenchymal stem cells (BMMSCs) remain the most widely used source of osteogenic cells in bone tissue engineering research. A cell-based treatment for alveolar ridge augmentation has received attention as an alternative to bone grafting. In the present study, BMMSC transplantation into tooth extraction sockets of $\mathrm{C} 57 \mathrm{BL} / 6 \mathrm{~J}$ mice was evaluated for alveolar ridge regeneration. The first right maxillary molars were extracted, and then BMMSCs (PDGFRa ${ }^{+}$Sca- $^{+}$CD45 $^{-}$TER119- $^{-}$cells) isolated from femoral and tibial bone marrow were immediately transplanted into the extraction sockets. A control group underwent the same procedure except for BMMSC transplantation. Bone formation in the sockets was evaluated using micro-computed tomography and histological and immunohistochemical analyses. At 3 weeks, bone formation in the sockets was more advanced in the experimental group than in the control group. Histological analysis at 6 weeks after transplantation showed that the sockets
\end{abstract}

Correspondence to Dr. Masaki Honda, Department of Oral Anatomy, Aichi-Gakuin University School of Dentistry, 1-100 Kusumoto-cho, Chikusa-ku, Nagoya, Aichi 464-8650, Japan Fax:+81-52-752-5988 E-mail: honda-m@dpc.agu.ac.jp

Color figures can be viewed in the online issue at J-STAGE. doi.org/10.2334/josnusd.18-0143

DN/JST.JSTAGE/josnusd/18-0143 in the experimental group also contained a greater quantity of bone marrow. Interestingly, socket bone mineral density was lower in the experimental group than in the control group at 6 weeks. These findings suggest that BMMSC transplantation accelerates bone healing and augments bone marrow formation in tooth extraction sockets.

Keywords: bone marrow; bone regeneration; dental socket; mesenchymal stem cells; tooth extraction.

\section{Introduction}

The alveolar ridge is a bony ridge on the maxilla or mandible, forming part of the alveolar process. Tooth extraction is a routine dental procedure frequently performed in cases of extensive caries, advanced periodontal disease and root fracture, or in particular cases to facilitate orthodontic treatment. After tooth extraction, the alveolar ridge undergoes uneven resorption and atrophy (1-5). Subsequent dimensional changes are more pronounced in the buccal wall than in its lingual/palatal counterparts, due to compromised blood supply and resorption of thin bundles of bone during healing $(3,4)$. These changes take place mainly in the first few months after extraction and continue during the following year, accounting for up to a $50 \%$ loss of pristine alveolar ridge width (6). Such changes during post-extraction 
alveolar ridge resorption result in bone volumes that are inadequate for placement of dental implants and also for providing stability for removable dentures. Regeneration at sites of bone loss is necessary for functional and esthetic optimization for placement of dental implants (7). Therefore, an understanding of the dynamics of extraction socket healing and the factors that determine successful ridge preservation is important for ensuring effective recovery from tooth extraction.

At present, the most widely accepted approaches for vertical and horizontal alveolar ridge augmentation $(8,9)$ and alveolar ridge/socket preservation (10) mainly involve the use of autogenous bone and/or bone substitute materials (11). Autogenous bone grafts from diverse origins are considered the gold standard for bone augmentation; however, their use is limited by the need for additional harvesting procedures and significant donor-site morbidity, and few outcome data are available (12-15).

Recently, tissue engineering and cell-based approaches have been identified as promising alternatives to autologous bone grafting for alveolar ridge augmentation (16). Tissue engineering generally refers to the harvesting of multipotent stem cells from an autologous source (e.g., bone marrow) and their subsequent in vitro culture and expansion to provide adequate numbers for regeneration of lost tissue (17). The applicability of cells in tissue engineering is dependent on two properties: self-renewal and pluripotency. Self-renewal refers to a cell's ability to go through numerous cycles of division while maintaining an undifferentiated state, whereas pluripotency refers to the capacity to differentiate into a wide range of mature cell types; both features are characteristic of embryonic stem cells. In postnatal life, stem cells are multipotent, which means they are able to differentiate into multiple, but limited, cell types. For example, bone marrow is one of the most applicable cell sources as it is a rich source of adult mesenchymal stem/progenitor cells.

Bone marrow-derived mesenchymal stem cells (BMMSCs) remain the most widely used source of osteogenic cells in bone-tissue engineering research, because they have a number of advantages for bone regeneration, including: (i) easy isolation and expansion in vitro, (ii) vigorous proliferation, (iii) their default pathway results in production of osteogenic cell types, and (iv) lack of ethical controversy associated with their use $(18,19)$. Furthermore, because BMMSCs have trophic activity that promotes vascularization and potent immunosuppressive effects, they have been the focus of a range of emerging therapeutics aimed at regeneration of damaged tissue and treatment of inflammation resulting from cardiovascular disease and myocardial infarction, brain and spinal cord injury, cartilage and bone injury, Crohn's disease, and graft-versus-host disease during bone marrow transplantation (20-23). However, their utilization in potential procedures for preventing dimensional changes in the alveolar bone after tooth extraction has received only limited attention. Hence, the objective of the present study was to evaluate the efficacy of BMMSC transplantation into fresh tooth extraction sockets for bone remodeling.

\section{Materials and Methods}

\section{Animals}

Eight- to 10-week-old C57BL/6J mice (Sankyo Laboratory, Tokyo, Japan) were used in this study. The mice were housed in an experimental animal room at $22^{\circ} \mathrm{C}$, with $55 \%$ relative humidity and a 12 -h light/dark cycle, and fed a standard laboratory diet. Water was provided ad libitum. All experiments were approved by the Animal Experimentation Committee of Nihon University School of Dentistry (AP16D032).

\section{Preparation of bone marrow-derived mesenchymal stromal cells}

The methods for isolation of BMMSCs used in this study have been described in detail previously (24). Briefly, the femurs and tibias of mice were dissected out and crushed using a mortar and pestle. The crushed bone fragments were digested in Dulbecco's modified Eagle medium (DMEM; Nacalai Tesque, Kyoto, Japan) (20 $\mathrm{mL}$ ) containing $0.2 \%$ collagenase (Wako, Tokyo, Japan). The resulting suspension was pelleted by centrifugation and the pellet resuspended for $5-10 \mathrm{~s}$ in water $(1 \mathrm{~mL})$ (Sigma Aldrich, St. Louis, MO, USA) to lyse the red blood cells. The remaining cells were then resuspended in Hanks balanced salt solution (Nacalai Tesque) and filtered through a cell strainer.

Cell sorting was performed using the following fluorescent label-conjugated antibodies: PE-conjugated CD45 (BD Biosciences, Franklin Lakes, NJ, USA) and TER119 (BD Biosciences), APC-conjugated platelet-derived growth factor receptor $\alpha$ (PDGFR $\alpha$; BD Biosciences), and FITC-conjugated stem cell antigen 1 (Sca-1; BD Biosciences), as described previously (24). The procedure was performed on a triple-laser MoFlo (Dako, Santa Clara, CA, USA) or FACScalibur (BD Biosciences) flow cytometer. After exclusion of hematopoietic $\left(\mathrm{CD} 45^{+}\right.$and TER119+) cells, one subpopulation in the $\mathrm{CD}^{+} 5^{-}$and TER $119^{-}$gated population was strongly positive for both PDGFR $\alpha^{+}$and Sca- $1^{+}$(Fig. 1A). The PDGFR $\alpha^{+}$and Sca- $1^{+}$cells were sorted as BMMSCs, which exhibited a 


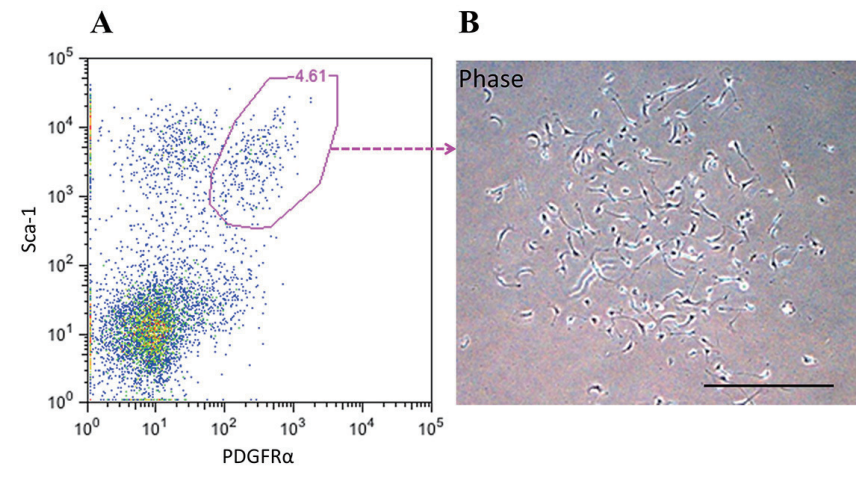

Fig. 1 Flow cytometric profile and morphology of BMMSCs.

A: One subpopulation (pink circles) were positive for both PDGFR $\alpha$ and Sca- 1 after gating of CD45 ${ }^{-}$and TER119 ${ }^{-}$cells.

B: PDGFR $\alpha^{+}$and $\mathrm{Sca}^{-1^{+}}$cells showed a spindle-shaped morphology. Scale bar: $100 \mu \mathrm{m}$.

typical spindle-shaped morphology and organization in a monolayer (Fig. 1B). These were selected for expansion and used in the following experiments.

\section{Extraction of teeth and BMMSC transplantation into dental sockets}

The first right maxillary molars of mice were extracted under anesthesia without causing fracture of the alveolar bone or tooth. For the experimental group $(n=5)$, BMMSCs $\left(4.0 \times 10^{4}\right.$ cells $/ 0.5 \mu \mathrm{L}$ of DMEM supplemented with $10 \%$ FBS and $1 \%$ penicillin/streptomycin) were immediately transplanted into the fresh extraction sockets (Fig. 2). After BMMSC transplantation, mice were left at rest for $1 \mathrm{~h}$, facing upward. The control group $(n=5)$ underwent tooth extraction without subsequent cell transplantation.

\section{Localization of fluorescently labeled BMMSCs}

BMMSCs were labeled with the fluorescent dye chloromethylbenzoamido 1,1'-dioctadecyl-3,3,3',3' tetramethylindocarbocyanine (DiI; Vybrant, V22885, Life Technologies, Eugene, OR, USA) in accordance with the manufacturer's recommendations. DiI-labeled cells were transplanted into the extraction sockets of the C57BL/6J mice. After 5 days, these mice were sacrificed and fluorescence microscopy (Biozero BZ-8000, Keyence, Osaka, Japan) was used to investigate the survival and localization of the transplanted cells.

\section{Micro-CT analysis}

Radiographic analysis by in vivo micro-computed tomography (micro-CT; Rigaku Co., Tokyo, Japan) of the extraction socket region was performed immediately following $(t=0)$ and at, 1, 2, 3, 4, 5, and 6 weeks after tooth extraction. Sagittal and 3-dimensional (occlusal
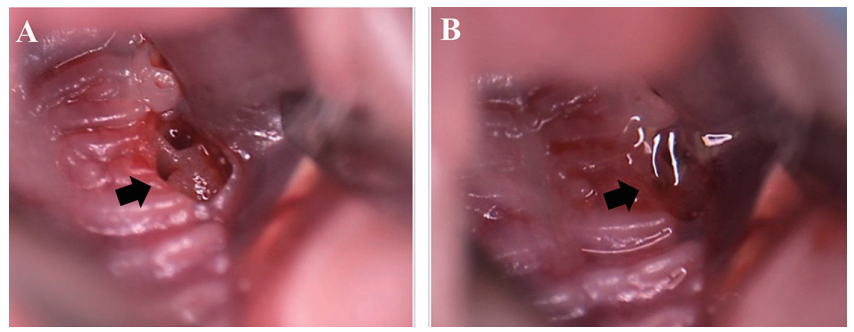

Fig. 2 Macroscopic intraoperative images of cell transplantation into the extraction socket.

A: The first right maxillary molars were extracted without bone or root fracture (black arrow).

B: PDGFR $\alpha^{+}$and Sca- $1^{+}$cells $\left(4.0 \times 10^{4}\right.$ cells $/ 0.5 \mu \mathrm{L}$ of culture medium) were immediately transplanted into each extraction socket (black arrows).

plane) images were examined to determine the level of bone tissue preservation. During exposure, mice were anesthetized with isoflurane (DS Pharma Animal Health Co. Ltd., Osaka, Japan). The exposure conditions were $17 \mathrm{~s}$ at $90 \mathrm{kV} / 100 \mathrm{~mA}$. The image volume consisted of a cylinder $2.4 \mathrm{~cm}$ in diameter and $2.4 \mathrm{~cm}$ long, with a voxel matrix size set to $481 \times 481 \times 483$ voxels. The bone volume and bone mineral density (BMD) in the sockets were measured in cubic millimeters $\left(\mathrm{mm}^{3}\right)$ and milligrams per cubic centimeter $\left(\mathrm{mg} / \mathrm{cm}^{3}\right)$ using quantitative analysis software (3by4 viewer 2011, Kitasenju Radist Dent, Tokyo, Japan). The measurement range was distributed between 3 roots - mesial, distal buccal, and distal lingual - and the upper and bottom parts of the formed bone were measured in each root. For the mesial root, the measurement ranges for the upper and bottom parts were defined as cylinders $2 \mathrm{~mm}$ in diameter and 0.5 $\mathrm{mm}$ in length, and $2 \mathrm{~mm}$ in diameter and $1 \mathrm{~mm}$ in length, respectively. For the distal buccal and distal lingual roots, the ranges for the upper and bottom parts were defined as cylinders $1.5 \mathrm{~mm}$ in diameter and $0.5 \mathrm{~mm}$ in length, and $1.5 \mathrm{~mm}$ in diameter and $1 \mathrm{~mm}$ in length, respectively.

\section{Histological and immunohistochemical analysis}

After micro-CT reconstruction, the surgical fields in the mandible for histological observation were resected at 6 weeks after transplantation. For each group, 5 tissue samples removed from 5 different mice were prepared. Extracted tissues were fixed overnight in $10 \%$ neutral buffered paraformaldehyde and incubated in $10 \%$ ethylenediaminetetraacetic acid (EDTA; Dojindo, Kumamoto, Japan) for 10 days at $4^{\circ} \mathrm{C}$. The tissues were then dehydrated in an ethanol series before embedding in paraffin. Paraffin-embedded tissues were cut into 8 - $\mu \mathrm{m}$-thick sections using a microtome and analyzed using hema- 


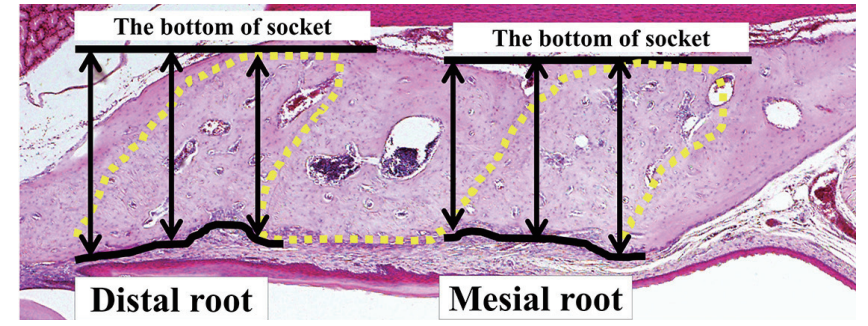

Fig. 3 Measurement of the height of newly formed bone in the extraction socket.

Perpendicular lines were drawn from the bottom of the socket to the surface of the newly formed bone at three points (at each side and the middle of the mesial root). The mean length of the three lines was calculated to determine the height of the mesial socket. The height at the distal socket was calculated using the same method.

toxylin and eosin (H-E) staining to compare bone tissue characteristics.

For histomorphometric analysis, $5 \mathrm{H}$-E-stained sections were selected per specimen, and the bone marrow area in the sockets was analyzed quantitatively using ImageJ software (National Institutes of Health, Bethesda, MD, USA). Measurement of height was carried out following the procedures shown in Fig. 3. First, three perpendicular lines were drawn from the bottom of the socket to the surface of the regenerated bone, marking each side and the middle of the mesial socket. Second, the mean length for the three lines was calculated and the height at the mesial root was defined. The height at the distal sockets was measured using the same method.

Tissue sections were deparaffinized, and tartrate-resistant acid phosphatase (TRAP) staining was performed using a commercial acid phosphatase leukocyte kit (Wako) to determine the localization of osteoclasts. In the newly formed bone, 5 randomly selected areas were observed with a light microscope at $\times 100$ magnification (Olympus, Tokyo, Japan) and the number of TRAP ${ }^{+}$cells in each area was counted.

To identify osteoblasts, the Runt-related transcription factor 2 (Runx2) was examined by immunohistochemistry. The sections were incubated overnight with an appropriate concentration of a primary antibody against human Runx2 (Abcam, Cambridge, UK). The slides were then exposed to Histofine Simple Stain Rat MAX PO MULTI (Nichirei Biosciences, Tokyo, Japan) containing 3,3'-diaminobenzidine (DAB) as a chromogen, to allow visualization of immune complexes.

To visualize proliferating cells, sections were stained for proliferating cell nuclear antigen (PCNA). Briefly, rabbit anti-human PCNA polyclonal antibody (Santa Cruz Biotech, Santa Cruz, CA, USA) was used in the primary reaction. The DAKO EnVision System, containing a
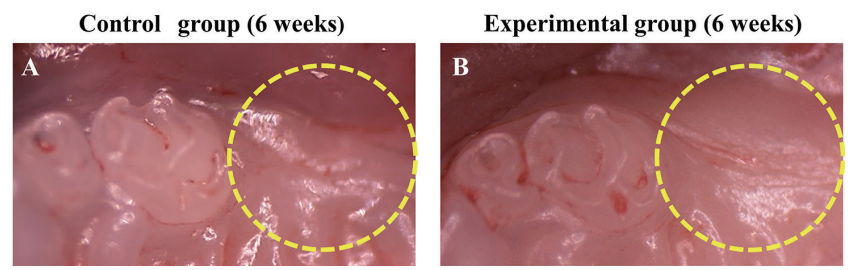

Fig. 4 Macroscopic appearance of the extraction socket at 6 weeks after BMMSC transplantation.

A: Control group. B: Experimental group. Yellow dotted lines represent the boundaries of the healed socket. Normal stratified squamous epithelium was observed in both groups.

secondary horseradish peroxidase-conjugated anti-rabbit antibody complex, was used in conjunction with DAB to detect PCNA. Histological and immunohistochemical examinations were performed using a light microscope equipped with a morphometric system (Olympus), connected to a personal computer.

\section{Statistical analysis}

Means and standard deviations were measured for bone volume, bone mineral density, bone marrow area, and the number of $\mathrm{TRAP}^{+}$cells in the extracted mandible tissue sections. Mann-Whitney $U$ test was used to analyze differences between two groups (experimental and control group) based on the micro-CT and histologic data. Differences at $P<0.05$ were considered statistically significant.

\section{Clinical findings}

\section{Results}

The macroscopic appearance of the socket at 6 weeks after transplantation is shown in Fig. 4. All sockets healed uneventfully with no signs of inflammation. There was a tendency for faster soft-tissue healing in the experimental group relative to the control group, although this difference was not statistically significant.

\section{Localization of fluorescently labeled BMMSCs in extraction sockets}

At 5 days after cell transplantation, DiI-labeled BMMSCs were easily visualized at the boundary between the gingival epithelium and lamina propria mucosa, suggesting that the transplanted cells had survived in the extraction socket up to this time. However, it was unclear whether the labeled cells were located in the gingival epithelium or the lamina propria mucosa (Fig. 5A, B). 


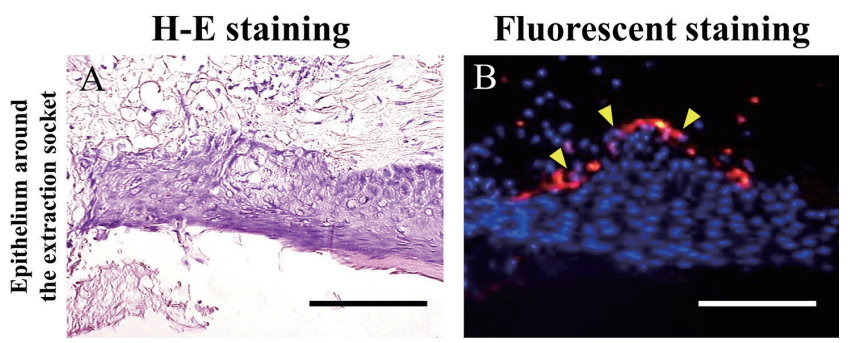

Fig. 5 Localization of transplanted cells using DiI fluorescence analysis.

A: H-E staining showed epithelialization and lamina propria regeneration, located directly below the gingival epithelium in the extraction socket at 5 days after BMMSC transplantation. Scale bar: $100 \mu \mathrm{m}$.

B: DiI-labeled cells (red) were seen at the boundary between the gingival epithelium and lamina propria mucosa in the extraction socket (yellow arrowheads). Blue-colored cells: DAPI. Scale bar: $100 \mu \mathrm{m}$.
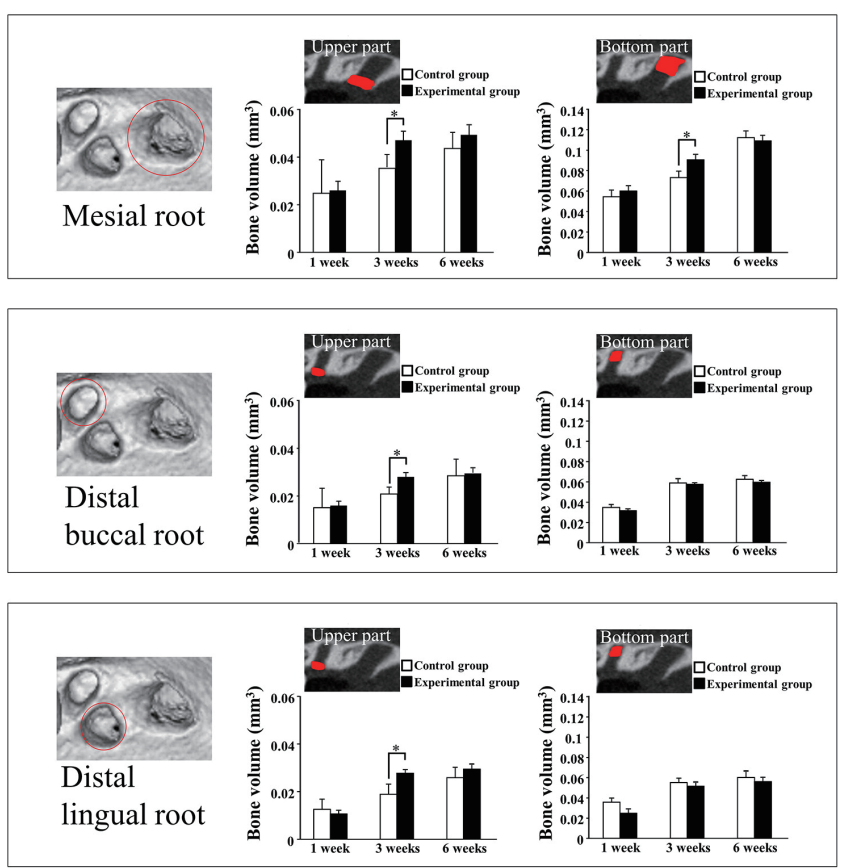

Fig. 7 Quantitation of newly formed bone volume in the extraction sockets of mesial, distal buccal, and distal lingual roots.

Upper section: bone volume of the upper and bottom mesial root. Middle section: bone volume in the upper and bottom distal buccal root. Lower section: bone volume in the upper and bottom distal lingual root. At 3 weeks, the bone volumes of all three upper roots were significantly higher in the experimental group than in the control group $(* P<0.05)$. In the bottom part, only the bone volume of the mesial root at 3 weeks was significantly higher relative to the control $(* P<0.05)$.

\section{Micro-CT analysis}

Representative processed micro-CT images (sagittal and occlusal planes) for each group are shown in Fig. 6. Sagittal plane images clearly showed that the extraction sockets were filled with newly formed bone up
A Control group

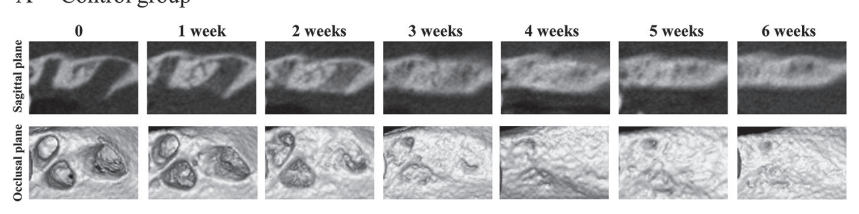

B Experimental group

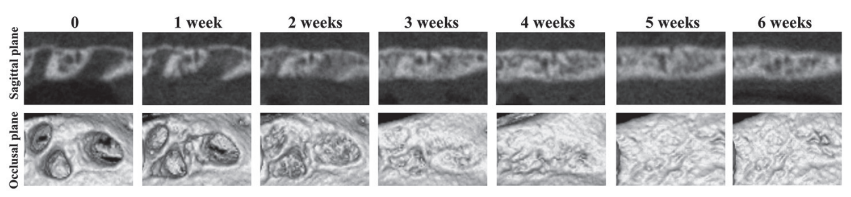

Fig. 6 Micro-CT analysis immediately $(t=0)$ and at 1, 2, 3, 4, 5, and 6 weeks after BMMSC transplantation.

Both groups showed gradually enhancing radiopacity of the extraction socket between 1 and 3 weeks. In the control group, radiopacity was most enhanced at 4 weeks, but no significant differences in radiopacity were evident between 4 and 6 weeks. Radiopacity reached maximum enhancement at 3 weeks in the experimental group.
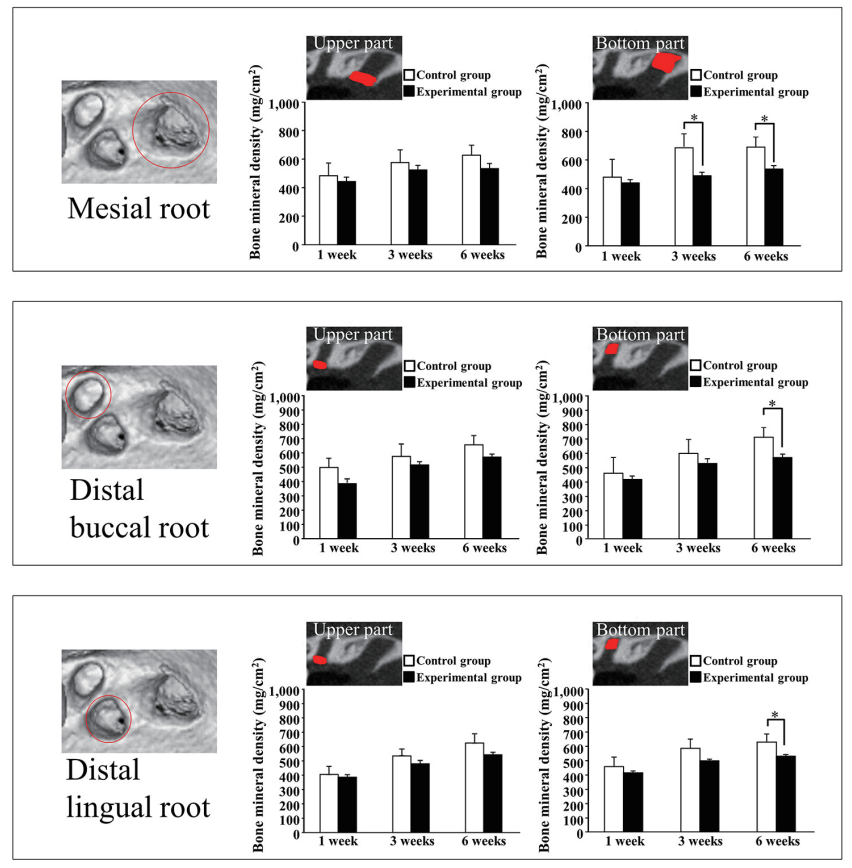

Fig. 8 Quantitation of newly formed bone mineral density (BMD) in the extraction socket of mesial, distal buccal, and distal lingual roots.

Upper section: BMD in the upper and bottom parts of the mesial root. Middle section: BMD in the upper and bottom distal buccal root. Lower section: BMD in the upper and bottom distal lingual root. After 3 weeks, the BMD of the bottom mesial root in the experimental group was significantly lower than that in the control group $(* P<0.05)$. After 6 weeks, the BMD of all three areas was significantly lower in the experimental group $(* P<0.05)$.

to the alveolar crest in both groups at 2 and 3 weeks, respectively. There was a tendency for an increase in radiopaque regions in the experimental group relative to the control group at both 2 and 3 weeks. Furthermore, occlusal plane images showed that the area of the bone 
H-E

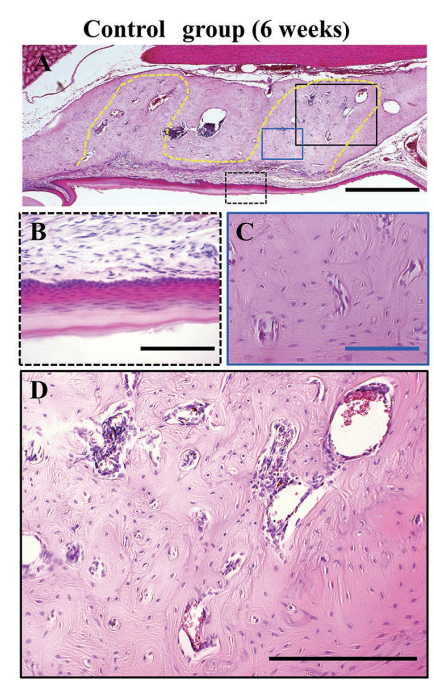

Experimental group (6 weeks)

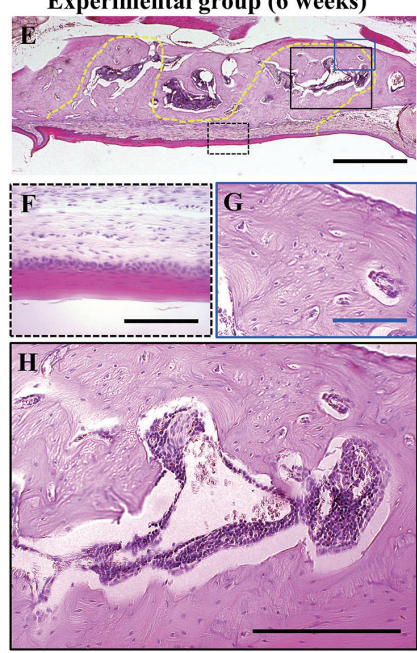

Fig. 9 Hematoxylin-eosin (H-E)-stained specimens of each sample at 6 weeks.

A-D: Control group. E-H: Experimental group. All images are representative of 5 independent experiments.

A, E: Transverse section showing the epithelialized extraction sockets filled with newly formed bone in both groups. It was difficult to differentiate between the socket edge (yellow dotted lines) and newly formed bone. Scale bars: $500 \mu \mathrm{m}$.

B, F: Higher magnification of boxed areas in Figs 7E and 9A (black dotted line in the mesial roots). Both groups showed stratified squamous epithelia. Scale bars: $50 \mu \mathrm{m}$.

C, G: Higher magnification of boxed areas in Figs 7E and 9A (blue line in the mesial roots). The newly formed bone in sockets was mature lamellar bone. Both groups showed woven bone with primary osteons and lamellar bone with secondary osteons. Scale bars: $50 \mu \mathrm{m}$

D, H: Higher magnification of boxed areas in Fig. 7A and E (black line in the mesial roots.). The experimental group had a larger amount of bone marrow in the sockets than the control group. Scale bars: $100 \mu \mathrm{m}$.

recess in extraction sockets was wider in the control group than in the experimental group within the followup period. Therefore, the bone volume in the upper and bottom areas of the extraction sockets were calculated for 3 extraction locations, i.e. the mesial, distal buccal, and distal lingual root sockets, using quantitative analysis software, as shown in Fig. 7. In the upper area, the bone volume for all 3 extraction sockets was significantly greater in the experimental group than in the control group at 3 weeks $(P<0.05)$. However, there were no significant differences between the groups at 6 weeks. In the bottom area, only the bone volume of the mesial root was significantly greater in the experimental group, and only at 3 weeks after extraction $(P<0.05)$.

Interestingly, the ratio of the transmission image area in the extraction sockets had a tendency to be retained between 3 and 6 weeks in the experimental group. At 6 weeks, the radiopaque area in the experimental
A
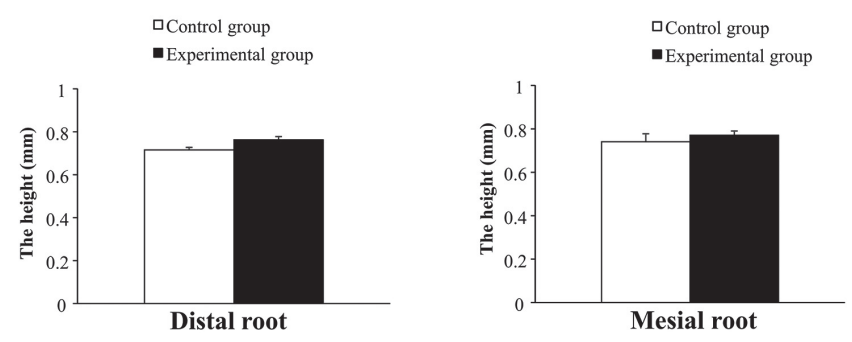

Fig. 10 Height of newly formed bone in the distal and mesial sockets.

The height of newly formed bone in the distal (A) and mesial (B) sockets did not differ significantly between the control and experimental groups $(* P<0.05)$.

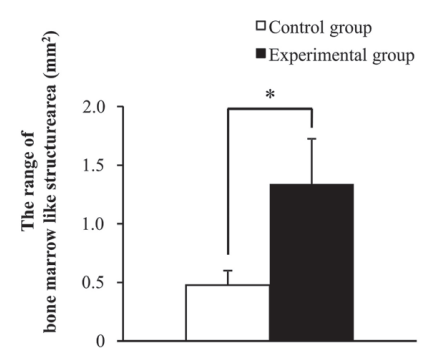

Fig. 11 Area of bone marrow in the extraction sockets at 6 weeks after transplantation.

The bone marrow area within the socket was significantly higher in the experimental group than in the control group $\left({ }^{*} P<0.05\right)$.

group seemed to be smaller than that the control group. Conversely, the radiopaque area in control group became gradually enhanced from 3 to 6 weeks. The BMD in these regions was calculated at 1,3 , and 6 weeks, and the results are summarized in Fig. 8. In the upper area, there were no significant differences in BMD between the groups within the follow-up period. However, BMD in the bottom area was significantly decreased in the experimental group in all extraction sockets from 6 weeks $(P<0.05)$.

\section{Histological and immunohistochemical analysis}

To identify new bone and bone marrow, histological evaluation with H-E staining was performed at 6 weeks after cell transplantation. Representative histological H-E-stained sections from each group are shown in Fig. 9. There was no evidence of inflammatory cell infiltration in any of the specimens. At this time, all extraction sockets had become epithelized and the lamina propria mucosae had regenerated, so that normal gingiva was evident in both groups. All of the samples exhibited well-organized and well-vascularized bone, with a lamellar architecture surrounding the Haversian canals. 
TRAP
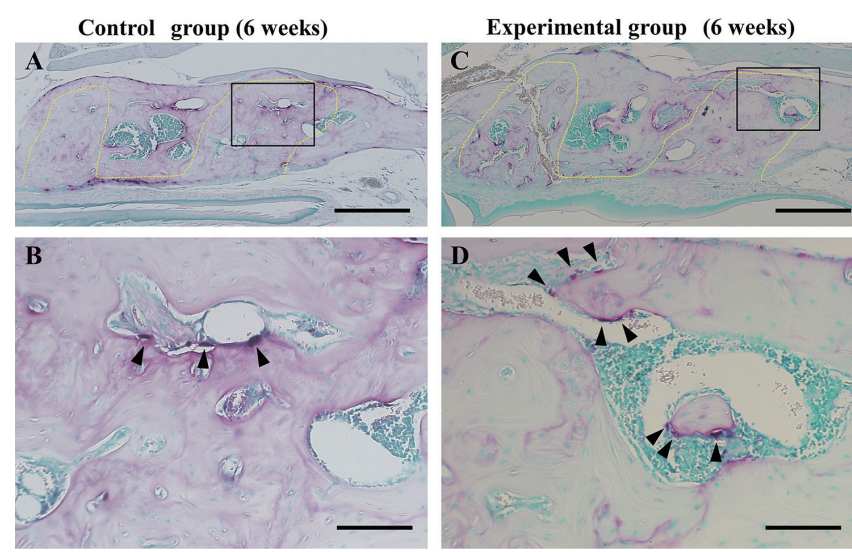

Fig. 12 Immunohistochemical staining for $\mathrm{TRAP}^{+}$cells in the extraction socket at 6 weeks.

A, C: Transverse section showing the extraction sockets. Yellow dotted lines indicate the socket edge. Scale bar: $500 \mu \mathrm{m}$.

$\mathrm{B}, \mathrm{D}$ : $\mathrm{TRAP}^{+}$osteoclasts are evident within the bone marrow of the sockets (black arrowheads). Scale bars: $100 \mu \mathrm{m}$.

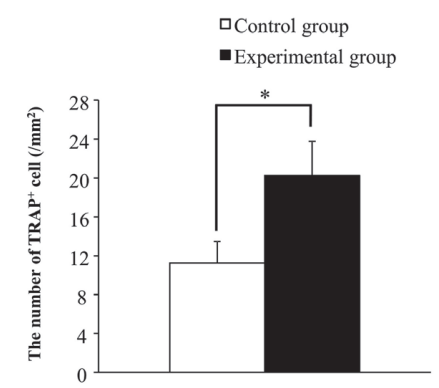

Fig. 13 Quantification of $\mathrm{TRAP}^{+}$osteoclasts in the extraction socket.

The number of $\mathrm{TRAP}^{+}$osteoclasts was significantly higher in the experimental group than in the control group in all bone marrow areas $(* P<0.05)$.

The alveolar bone heights of the distal and mesial extraction sockets were measured from H-E-stained sections, and no significant differences between the experimental and control groups were evident (Fig. 10). However, the sockets were occupied by mineralized bone and bone marrow at various degrees of maturation, depending on the extent of healing in each group (Fig. 9A, E: yellow dotted lines). At high magnification, normal stratified squamous epithelium was observed in both groups (Fig. $9 \mathrm{~B}, \mathrm{~F})$ and the hard tissue was composed of woven bone with primary osteons and lamellar bone with secondary osteons (Fig. 9C, G). Interestingly, the sockets in the experimental group contained a greater quantity of bone marrow than those in the control group (Fig. 9D, H), and showed a significant increase in bone marrow area (Fig. 11), consistent with observations of corresponding micro-CT images. To determine the localization of osteo-

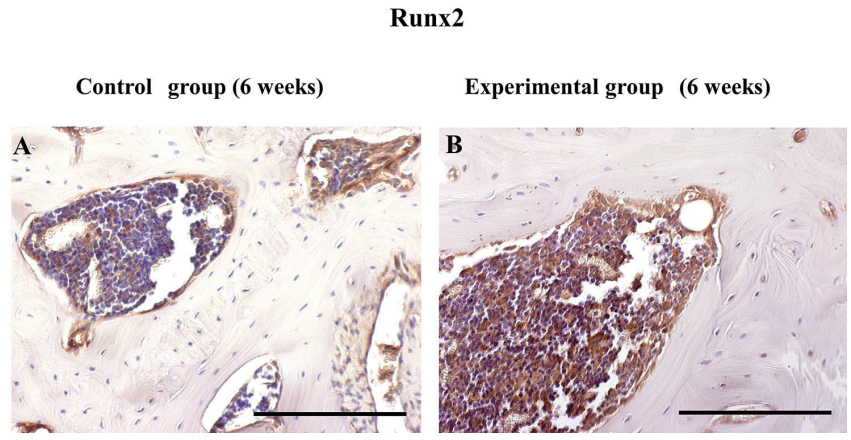

Fig. 14 Immunohistochemical staining for Runx $2^{+}$cells in the extraction socket at 6 weeks.

A, B: Both groups showed a large number of Runx $2^{+}$osteoblasts at the surface of the existing bone, particularly within the socket bone marrow. Scale bars: $100 \mu \mathrm{m}$.

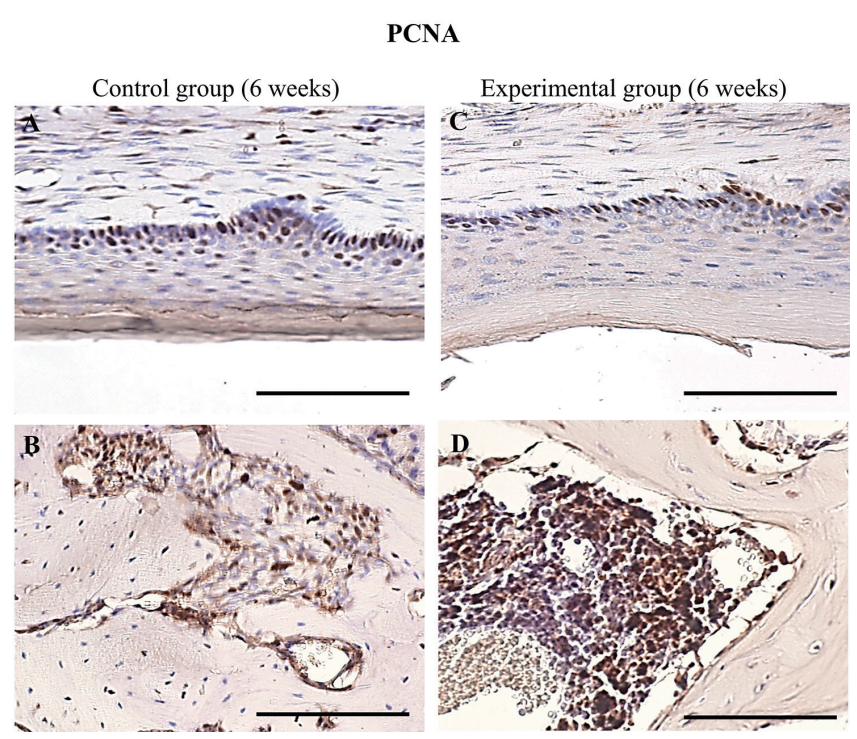

Fig. 15 Immunohistochemical staining for $\mathrm{PCNA}^{+}$cells in the extraction socket at 6 weeks.

A-D: $\mathrm{PCNA}^{+}$cells were observed in the basement membrane of the epithelia in both groups. These cells were widely observed in the bone marrow, but were not evident in the bone area of the socket. Scale bar: $100 \mu \mathrm{m}$.

clasts, immunohistochemical staining and quantitative analysis of $\mathrm{TRAP}^{+}$cells were performed. $\mathrm{TRAP}^{+}$cells were located mainly within the interface zone of the bone cavity, including the bone marrow (Fig. 12, black arrowheads). Furthermore, the number of $\mathrm{TRAP}^{+}$osteoclasts was significantly higher in the experimental group than in the control group (Fig. 13; $P<0.05$ ). However, the localization of Runx $2^{+}$and $\mathrm{PCNA}^{+}$cells was similar in the two groups (Figs. 14, 15).

\section{Discussion}

Cell transplantation is a promising new approach for treatments focusing on tissue regeneration. Although 
investigations of combined utilization of BMMSCs and engineered tissue scaffolds for alveolar bone preservation have been limited (25), no previous studies have investigated transplantation of BMMSCs without scaffolds. Hence, the objective of the present study was to evaluate the effectiveness of scaffold-free BMMSC transplantation into tooth extraction sockets for bone and bone marrow regeneration, with the aim of establishing a minimally invasive technique for reconstruction of the alveolar ridge following tooth extraction.

The bone regeneration process that follows tooth extraction is an interesting experimental model for studies of bone repair, which includes the overlapping phases of inflammation, granulation tissue formation, angiogenesis, and extracellular matrix deposition. The role of BMMSCs in the wound healing process has already been studied extensively. Macroscopic observation in this study revealed that granulation tissue formation and epithelialization in the extraction socket were accelerated by BMMSC transplantation, thus supporting previous findings (26).

Tissue regeneration is mediated by various factors, including cytokines and growth factors, which are secreted from transplanted mesenchymal stem cells, such as BMMSCs and adipose-derived mesenchymal stem cells (26). Hence, the survival of transplanted cells is an important factor in determining the rate of tissue regeneration $(26,27)$. The present results also showed that fluorescently labeled transplanted cells were able to survive in extraction sockets for at least 5 days following transplantation.

When bone regeneration levels were compared using micro-CT, the experimental group exhibited a significantly greater area of newly formed bone in comparison with the control group at both 2 and 3 weeks after transplantation. Interestingly, micro-CT analysis showed that the radiopaque area in experimental extraction sockets was maintained from 3 to 6 weeks after BMMSC transplantation. In contrast, the radiopaque area in the control group gradually decreased during the same time period. Previous reports have suggested that the process of mineralization differs depending on the location within the extraction socket (28). For this reason, the present study evaluated the difference between the upper and bottom parts of the extraction socket. In the upper part, there was a significant difference in the volume of newly formed bone in all three roots between the control and experimental groups at 3 weeks, but no difference at 1 and 6 weeks, although the vertical distances of the distal and mesial crests did not differ significantly. Interestingly, the results were not consistent with those of previous studies, which implied that bone formation was initiated from the bottom region (28-30). As transplanted cells were detected at the boundary between the gingival epithelium and lamina propria mucosa, the upper part of the extraction socket appeared to show greater bone regeneration. The position of this boundary coincides with the position of the upper part, and thus the majority of transplanted cells were located in this region. Therefore it is suggested that transplanted cells located in the upper extraction socket may enhance early mineralization in this region for up to 3 weeks after transplantation.

The close association between osteogenesis and angiogenesis plays a key role in the process of bone repair (31); bone marrow appears to be an important source of mobilized progenitor/stem cells involved in not only vasculogenesis (the formation of new blood vessels from endothelial progenitor cells [EPCs]), but also formation of new blood vessels via angiogenesis (32). Bone marrowderived EPCs directly form new vessels and are a rich source of pro-angiogenic factors $(33,34)$. At 6 weeks, all 3 root sockets in the control group showed significantly higher BMD than those in the experimental group. To clarify the reason for this difference, bone marrow in extraction sockets was analyzed histologically. At 6 weeks, the area of regenerated bone marrow after cell transplantation was clearly greater in the experimental group than in the control group.

The present study did not investigate in detail the mechanism by which transplanted cells can enhance bone regeneration. However, many previous reports have confirmed that BMMSCs exert their regenerative function via cytokine secretion (27). For example, BMMSC transplantation has previously been shown to increase the local expression of vascular endothelial growth factor (VEGF). Angiogenesis, which is initiated by cytokines such as VEGF, plays an important role in bone regeneration. Hypoxia-induced increases in VEGF concentration stimulate endothelial cell mitosis and angiogenesis, thereby improving the oxygen supply $(35,36)$. Based on these assumptions, further study is required to clarify the mechanism involved in bone healing at tooth extraction sites after BMMSC transplantation. In dentistry, dry socket is a common and severe condition associated with a high morbidity rate. However, the outcomes of existing conservative treatments are uncertain. Although the pathogenetic mechanism responsible for dry socket remains unclear, it is known that poor blood supply to the extraction socket leads to eventual bone necrosis. Therefore, rebuilding and improving the supply of blood to the extraction socket has been proposed as an effective therapeutic approach. Bone marrow, in addition to its 
crucial role in hematopoiesis, is involved in the process of vasculogenesis (37). Accordingly, BMMSC transplantation may have the potential to prevent necrosis in dry sockets.

As recruitment of osteoclasts is known to be an important factor involved in bone homeostasis $(38,39)$, the generation of $\mathrm{TRAP}^{+}$osteoclasts was next examined. Significant differences in the numbers of $\mathrm{TRAP}^{+}$cells were observed between the experimental and control groups, suggesting that BMMSC transplantation into extraction sockets is effective for generating high numbers of osteoclasts. Bone homeostasis relies on the balance between osteoblasts, which are responsible for new bone matrix formation, and osteoclasts, which regulate bone matrix decomposition and calcium reuptake and are therefore important for bone remodeling. Hence, BMMSC transplantation may be effective for not only generating new bone marrow, but also maintaining bone homeostasis.

Previous results have suggested that delayed healing may occur at sites where tissue has been grafted to assist ridge preservation $(40,41)$. De novo contact osteogenesis around a graft probably occurs more slowly than osteogenesis distant from extraction socket walls, because the migration of osteogenic cells from that location must be preceded by osteoconduction (42). Therefore, scaffolds without osteoconduction may not be necessary to support mesenchymal stem cells, such as BMMSCs, to maintain bone homeostasis in the tooth socket, as this process involves distant osteogenesis.

The main limitation of the present study was the short time period following transplantation during which the analysis was conducted. This period may have been insufficient for predicting long-term responses to BMMSC transplantation. This study was also conducted using a relatively small sample size, and therefore additional experiments using larger study cohorts will be required.

In conclusion, within the limitations of this study, BMMSC transplantation may result in higher rates of bone marrow regeneration in the extraction socket and significantly accelerate bone maturation. It is also suggested that BMMSCs play an important role in bone homeostasis within the extraction socket, and this significant finding requires further investigation.

\section{Acknowledgements}

This work was supported in part by JSPS KAKENHI Grant Numbers JP15H05037, JP15K15274, JP15K11323, JP15K20562, Dental Research Center, Nihon University School of Dentistry, and the Sato Fund, Nihon University School of Dentistry.

\section{Conflict of interest}

The authors have no conflict of interest to declare.

\section{References}

1. Devlin H, Ferguson MW (1991) Alveolar ridge resorption and mandibular atrophy. A review of the role of local and systemic factors. Br Dent J 170, 101-104.

2. Lekovic V, Camargo PM, Klokkevold PR, Weinlaender M, Kenney EB, Dimitrijevic B et al. (1998) Preservation of alveolar bone in extraction sockets using bioabsorbable membranes. J Periodontol 69, 1044-1049.

3. Cardaropoli G, Araujo M, Lindhe J (2003) Dynamics of bone tissue formation in tooth extraction sites. An experimental study in dogs. J Clin Periodontol 30, 809-818.

4. Araujo MG, Lindhe J (2005) Dimensional ridge alterations following tooth extraction. An experimental study in the dog. J Clin Periodontol 32, 212-218.

5. Barone A, Aldini NN, Fini M, Giardino R, Calvo Guirado JL, Covani U (2008) Xenograft versus extraction alone for ridge preservation after tooth removal: a clinical and histomorphometric study. J Periodontol 79, 1370-1377.

6. Schropp L, Wenzel A, Kostopoulos L, Karring T (2003) Bone healing and soft tissue contour changes following single-tooth extraction: a clinical and radiographic 12-month prospective study. Int J Periodontics Restorative Dent 23, 313-323.

7. Chiapasco M, Casentini P, Zaniboni M (2009) Bone augmentation procedures in implant dentistry. Int J Oral Maxillofac Implants 24, 237-259.

8. Tonetti MS, Hammerle CH, European Workshop on Periodontology Group C (2008) Advances in bone augmentation to enable dental implant placement: consensus report of the sixth European workshop on periodontology. J Clin Periodontol 35, 168-172.

9. Esposito M, Grusovin MG, Felice P, Karatzopoulos G, Worthington HV, Coulthard P (2009) Interventions for replacing missing teeth: horizontal and vertical bone augmentation techniques for dental implant treatment. Cochrane Database Syst Rev 2009 Oct 7, doi: 10.1002/14651858. CD003607.pub4.

10. Horvath A, Mardas N, Mezzomo LA, Needleman IG, Donos N (2013) Alveolar ridge preservation. A systematic review. Clin Oral Investig 17, 341-363.

11. Jensen SS, Terheyden H (2009) Bone augmentation procedures in localized defects in the alveolar ridge: clinical results with different bone grafts and bone-substitute materials. Int J Oral Maxillofac Implants 24, 218-236.

12. Becker BE, Becker W, Ricci A, Geurs N (1998) A prospective clinical trial of endosseous screw-shaped implants placed at the time of tooth extraction without augmentation. J Periodontol 69, 920-926.

13. Nevins M, Camelo M, De Paoli S, Friedland B, Schenk RK, Parma-Benfenati S et al. (2006) A study of the fate of the buccal wall of extraction sockets of teeth with prominent roots. Int J Periodontics Restorative Dent 26, 19-29. 
14. Fickl S, Zuhr O, Wachtel H, Bolz W, Huerzeler M (2008) Tissue alterations after tooth extraction with and without surgical trauma: a volumetric study in the beagle dog. J Clin Periodontol 35, 356-363.

15. Nkenke E, Stelzle F (2009) Clinical outcomes of sinus floor augmentation for implant placement using autogenous bone or bone substitutes: a systematic review. Clin Oral Implants Res 20, 124-133.

16. Khojasteh A, Behnia H, Dashti SG, Stevens M (2012) Current trends in mesenchymal stem cell application in bone augmentation: a review of the literature. J Oral Maxillofac Surg 70, 972-982.

17. Egusa H, Sonoyama W, Nishimura M, Atsuta I, Akiyama K (2012) Stem cells in dentistry--part II: clinical applications. J Prosthodont Res 56, 229-248.

18. Toma C, Pittenger MF, Cahill KS, Byrne BJ, Kessler PD (2002) Human mesenchymal stem cells differentiate to a cardiomyocyte phenotype in the adult murine heart. Circulation 105, 93-98.

19. Sasaki M, Abe R, Fujita Y, Ando S, Inokuma D, Shimizu H (2008) Mesenchymal stem cells are recruited into wounded skin and contribute to wound repair by transdifferentiation into multiple skin cell type. J Immunol 180, 2581-2587.

20. Martens TP, See F, Schuster MD, Sondermeijer HP, Hefti MM, Zannettino A et al. (2006) Mesenchymal lineage precursor cells induce vascular network formation in ischemic myocardium. Nat Clin Pract Cardiovasc Med 3, S18-22.

21. Fibbe WE, Nauta AJ, Roelofs H (2007) Modulation of immune responses by mesenchymal stem cells. Ann N Y Acad Sci 1106, 272-278.

22. Nauta AJ, Fibbe WE (2007) Immunomodulatory properties of mesenchymal stromal cells. Blood 110, 3499-3506.

23. Phinney DG, Prockop DJ (2007) Concise review: mesenchymal stem/multipotent stromal cells: the state of transdifferentiation and modes of tissue repair--current views. Stem Cells 25, 2896-2902.

24. Houlihan DD, Mabuchi Y, Morikawa S, Niibe K, Araki D, Suzuki S et al. (2012) Isolation of mouse mesenchymal stem cells on the basis of expression of Sca-1 and PDGFR-alpha. Nat Protoc 7, 2103-2111.

25. De Kok IJ, Drapeau SJ, Young R, Cooper LF (2005) Evaluation of mesenchymal stem cells following implantation in alveolar sockets: a canine safety study. Int J Oral Maxillofac Implants 20, 511-518.

26. Werner S, Grose R (2003) Regulation of wound healing by growth factors and cytokines. Physiol Rev 83, 835-870.

27. Noiseux N, Gnecchi M, Lopez-Ilasaca M, Zhang L, Solomon SD, Deb A et al. (2006) Mesenchymal stem cells overexpressing Akt dramatically repair infarcted myocardium and improve cardiac function despite infrequent cellular fusion or differentiation. Mol Ther 14, 840-850.

28. Amler MH (1969) The time sequence of tissue regeneration in human extraction wounds. Oral Surg Oral Med Oral Pathol 27, 309-318.
29. Heberer S, Al-Chawaf B, Hildebrand D, Nelson JJ, Nelson K (2008) Histomorphometric analysis of extraction sockets augmented with Bio-Oss Collagen after a 6-week healing period: a prospective study. Clin Oral Implants Res 19, 12191225 .

30. Trombelli L, Farina R, Marzola A, Bozzi L, Liljenberg B, Lindhe J (2008) Modeling and remodeling of human extraction sockets. J Clin Periodontol 35, 630-639.

31. Kanczler JM, Oreffo RO (2008) Osteogenesis and angiogenesis: the potential for engineering bone. Eur Cell Mater 15, 100-114.

32. Carmeliet P (2003) Angiogenesis in health and disease. Nat Med 9, 653-660.

33. Rehman J, Li J, Orschell CM, March KL (2003) Peripheral blood "endothelial progenitor cells" are derived from monocyte/macrophages and secrete angiogenic growth factors. Circulation 107, 1164-1169.

34. Thijssen DH, Torella D, Hopman MT, Ellison GM (2009) The role of endothelial progenitor and cardiac stem cells in the cardiovascular adaptations to age and exercise. Front Biosci (Landmark Ed) 14, 4685-4702.

35. Tang TT, Lu B, Yue B, Xie XH, Xie YZ, Dai KR et al. (2007) Treatment of osteonecrosis of the femoral head with hBMP2-gene-modified tissue-engineered bone in goats. J Bone Joint Surg Br 89, 127-129.

36. Hung SC, Pochampally RR, Chen SC, Hsu SC, Prockop DJ (2007) Angiogenic effects of human multipotent stromal cell conditioned medium activate the PI3K-Akt pathway in hypoxic endothelial cells to inhibit apoptosis, increase survival, and stimulate angiogenesis. Stem cells 25, 23632370 .

37. Carmeliet P (2000) Mechanisms of angiogenesis and arteriogenesis. Nat Med, 389-395.

38. Kondo N, Ogose A, Tokunaga K, Umezu H, Arai K, Kudo N et al. (2006) Osteoinduction with highly purified beta-tricalcium phosphate in dog dorsal muscles and the proliferation of osteoclasts before heterotopic bone formation. Biomaterials 27, 4419-4427.

39. Davison NL, Gamblin AL, Layrolle P, Yuan H, de Bruijn JD, Barrere-de Groot F (2014) Liposomal clodronate inhibition of osteoclastogenesis and osteoinduction by submicrostructured beta-tricalcium phosphate. Biomaterials 35, 5088-5097.

40. Hong JY, Lee JS, Pang EK, Jung UW, Choi SH, Kim CK (2014) Impact of different synthetic bone fillers on healing of extraction sockets: an experimental study in dogs. Clin Oral Implants Res 25, e30-37.

41. Lindhe J, Cecchinato D, Donati M, Tomasi C, Liljenberg B (2014) Ridge preservation with the use of deproteinized bovine bone mineral. Clin Oral Implants Res 25, 786-790.

42. Kim JJ, Schwarz F, Song HY, Choi Y, Kang KR, Koo KT (2017) Ridge preservation of extraction sockets with chronic pathology using Bio-Oss ${ }^{\circledR}$ Collagen with or without collagen membrane: an experimental study in dogs. Clin Oral Implants Res 28, 727-733. 